\title{
Validation study of Sinhala version of the dermatology life quality index (DLQI)
}

\author{
N P Madarasingha ${ }^{1}$, P de Silva ${ }^{2}$, K Satgurunathan ${ }^{1}$ \\ (Index words: quality of life, dermatological disease, validation)
}

\begin{abstract}
Introduction The effect of dermatological disease on patients' quality of life is a minimally addressed issue in Sri Lanka. One reason is the unavailability of a standardised measuring tool. This study was done to produce and validate a Sinhala version of the Dermatology Life Quality Index (DLQI), which is an internationally accepted quality of life measuring instrument.

Methods The English version of the DLQI was translated according to standard procedures and the translated version was validated among 200 outpatients attending the dermatology clinics at the National Hospital of Sri Lanka and 30 healthy volunteers. The SF-36 was administered simultaneously to a random subsample
\end{abstract}

of 125 participants to test for criterion validity. Internal consistency and the test-retest reliability were assessed separately for the translated DLQI.

Results The Sinhala version of DLQI takes 3-5 minutes to be completed on average and had no confusing, embarrassing or difficult to answer questions. Except for personal relationship domain (0.442) all other domains demonstrated high Cronbach's alpha values suggesting satisfactory internal consistency within the domains. The DLQI demonstrated good test-retest reliability with all Kappa values above 0.83 . In comparison with the relevant domains of the SF-36, the DLQI demonstrated greater than 0.4 (Pearson r) suggesting an acceptable criterion validity. The Sinhala version of the DLQI appears to discriminate well between disease status and disease severity $(\mathrm{F}=31.09, p<0.001, \mathrm{n}=240)$.

${ }^{1}$ Dermatology Unit, National Hospital of Sri Lanka and ${ }^{2}$ National Institute of Health Sciences, Kalutara, Sri Lanka. Correspondence: NPM, e-mail <nayanimadara@yahoo.com>. Received 15 February and revised version accepted 9 October 2010. Competing interests: none declared. 
Conclusions The DLQI (Sinhala) version is a simple, acceptable and reliable tool to measure the effect of dermatological diseases on quality of life.

Ceylon Medical Journal 2011; 56: 18-22

\section{Introduction}

Skin diseases and their treatment cause psychological distress to patients and impair their quality of life $[1,2]$. Although addressed in developed countries the Quality of Life (QoL) aspect is often overlooked in Sri Lanka [3,4]. One of the main drawbacks we have is the unavailability of a standardised measuring tool for assessing the quality of life among patients.

The Dermatology Life Quality Index (DLQI) questionnaire was first described in 1994 [5]. At present it is being used for 33 different skin conditions in 32 countries and is available in 55 languages including Tamil. DLQI is the most frequently used instrument in studies of randomised controlled trials, for assessing the outcome [6]. Therefore the translation of DLQI to Sinhala and a validation study to assess its cultural acceptability, reliability and validity was done.

\section{Methods}

Consent for the translation process was obtained from the copyright holders of DLQI. Two independent translators translated the DLQI from English to Sinhala. The final combined translation was backtranslated to English by a bilingual person. This backtranslated version was sent to the original authors and modifications were done with the discussion of the two parties.

The DLQI consists of 10 questions covering various aspects of quality of life. The DLQI was calculated by simple summation of the score of each question resulting in a maximum of 30 and a minimum of 0 . The scoring and interpretation of incorrectly marked questions were done according to the standards given by the original authors. Approval for the study was obtained from the Ethical Review Committee of the National Hospital of Sri Lanka.

Sinhala-literate outpatients over 16 years, who were attending the dermatology clinics of the National Hospital of Sri Lanka were included in the study. The patients were given a brief introduction of the purpose of the questionnaire and verbal consent was taken prior to the commencement of the DLQI administration.

The validity was assessed employing several methods. Face and content validity had already been established for the DLQI by its original author and the validity of the translated version was assessed by a panel of experts. Each item in the instrument was assessed for its relevance in assessing quality of life among dermatological patients, appropriateness of the wording and acceptability in the local context.
Reliability of the DLQI was assessed by means of internal consistency within the domains and assessing test-retest reliability. Internal consistency is the extent to which items within a domain are correlated with each other to give the same result. This was assessed by calculating the Cronbach's alpha coefficient. Estimates of a magnitude of 0.70 or greater was considered as satisfactory [8].

Test-retest reliability measures the ability to generate stable results which are reproducible [9]. This was assessed by re-administering the DLQI after two weeks to a sub sample of 20 patients selected randomly. A correlation coefficient (Cohen's Kappa) of 0.60 or above was considered a good level of agreement between scores [9].

Acceptability was assessed by the response rate, percentage of incorrectly completed questionnaires, the time required to complete the questionnaire and details of items considered upsetting, confusing or difficult by the respondents.

DLQI scores were compared among healthy and diseased groups as well as among groups with varied severity of the disease to assess the discriminant validity. Criterion validity was assessed by comparison with selected components of the translated and validated version of the SF-36 instrument (regarding role-physical, role-emotional, social functioning, mental health, and vitality). A few questions from SF-36 which were thought to be most relevant to patients with skin diseases were selected and was used in a randomly selected sub sample of 125 patients. A moderate correlation (Pearson $\mathrm{r}=0.40$ 0.70 ) between the DLQI and the SF-36 components was considered to be adequate [7].

\section{Results}

The backtranslated version of DLQI (Sinhala) was accepted by the original authors except for question 8 . The back translation denoted the term 'spouse' to the word which appeared as 'partner' in the original English version. As the word partner in its meaning in the western culture is not familiar to the local context ultimately it was decided to go ahead with the same word which is more relevant for the local community and the translated version was accepted for the validation.

This translated Sinhala version of the DLQI is available with the copyright owners at http://www. dermatology.org.uk/quality/quality-dlqi.html under different language versions. This can be downloaded for any future use in Sri Lanka with the permission of the original authors.

The DLQI (Sinhala) was tested on 200 outpatients with various dermatological problems. The mean age of the study population was 40.3 years with nearly equal sex distribution.

Distribution of the study sample based on the disease is illustrated in Table 1. 
Table 1. Distribution of the study sample based on the disease

\begin{tabular}{lcc}
\hline Disease & No. & Percentage \\
\hline Eczema & 49 & 24.5 \\
Psoriasis & 46 & 23.0 \\
Acne & 20 & 10.0 \\
Vitiligo & 29 & 14.5 \\
Infections & 21 & 10.5 \\
Other & 35 & 17.5 \\
\hline
\end{tabular}

In assessing the content validity, colloquialization of few words were required. Additionally, the appropriateness of question number 6 in the local context, which was regarding sports, was questioned.

Reliability of the translated DLQI as assessed by measuring the internal consistency (Cronbach's alpha) is presented in Table 2, separately for the healthy volunteers and the patients attending the clinics.

Table 2. Distribution of coefficient alphas for the domains of the DLQI (Sinhala)

\begin{tabular}{lccc}
\hline DLQI domain & $\begin{array}{c}\text { No. of } \\
\text { items }\end{array}$ & $\begin{array}{c}\text { Healthy } \\
\text { volunteers } \\
(\mathrm{n}=40)\end{array}$ & $\begin{array}{c}\text { Patients } \\
(\mathrm{n}=200)\end{array}$ \\
\hline Symptoms and feelings & 2 & .598 & .561 \\
Daily activities & 2 & .654 & .741 \\
Leisure & 2 & .569 & .687 \\
Personal relationships & 2 & .498 & .442 \\
\hline
\end{tabular}

Work and school domains and the treatment domain had only one question and were excluded in assessing internal consistency. Except for the personal relationship domain, all other domains indicate a satisfactory level of internal consistency with Cronbach's alpha values ranging from 0.561 to 0.741 . Although quality of life is a variable phenomenon, re-administering the DLQI after two weeks to a subsample of 20 patients selected randomly showed a test-retest reliability coefficient of 0.83 , where any correlation greater than 0.70 was considered as satisfactory. The comparison of the DLQI scores among the healthy and diseased groups was used to assess the discriminant validity. Healthy and diseased groups as well as severity of the disease within the diseased groups were compared separately. The statistical significance of the observed differences was assessed using the ANOVA test (see Tables 3 and 4).

Table 3. Distribution of the DLQI in relation to disease category

\begin{tabular}{lcccccc}
\hline Disease & Mean DLQI score & Number & Minimum & Maximum & F value & $p$ value \\
\hline Eczema & 8.94 & 49 & 1 & 24 & & \\
Psoriasis & 10.54 & 46 & 2 & 25 & & \\
Acne & 7.95 & 20 & 1 & 23 & 2.425 & \\
Vitiligo & 7.55 & 29 & 1 & 22 & $\mathrm{df}=234$ & 0.037 \\
Infections & 5.90 & 21 & 0 & 20 & & \\
Other & 8.34 & 35 & 1 & 19 & & \\
\hline
\end{tabular}


Table 4. Distribution of DLQI in relation to disease status and severity of disease

\begin{tabular}{lccccc}
\hline Disease severity & $n$ & Mean DLQI & Range & $F$ & $d f$ \\
\hline Healthy volunteers & 40 & 0.70 & $0.36-1.04$ & \\
Mild & 54 & 7.33 & $5.68-8.98$ & $\mathrm{~F}=31.09$ \\
Moderate & 117 & 8.44 & $7.49-9.40$ & $\mathrm{df}=236$ \\
Severe & 29 & 11.48 & $9.30-13.67$ & $\mathrm{p}<.001$ \\
\hline
\end{tabular}

Table 5. Correlations between scores in SF-36 and DLQI (Sinhala)

\begin{tabular}{llll}
\hline SF 36 Domains & DLQI (Sinhala) & $N$ & $P$ \\
\hline Role - physical & -0.639 & 125 & $<0.01$ \\
Vitality & -0.499 & 125 & $<0.01$ \\
Mental health & -0.579 & 125 & $<0.01$ \\
Social functioning & -0.645 & 125 & $<0.01$ \\
Role-emotional & -0.541 & 125 & $<0.01$ \\
\hline
\end{tabular}

Overall, these findings suggest satisfactory discriminant validity for the translated Sinhala version of the DLQI. Results pertaining to the criterion validity assessed for the Sinhala version of the DLQI in relation to the relevant components of the validated Sinhalese version of SF-36, are presented in table 5.

If a moderate correlation of above 0.4 was considered is satisfactory, the item correlations demonstrated by the DLQI (Sinhala) can be considered satisfactory and having good criterion validity.

The response rate for the DLQI was around 98\%. The rate of successful completion of scale items was in excess of $95 \%$ in all items other than item 7 . This question was misunderstood by $12 \%$. The time taken to complete the DLQI ranged from 3-5 minutes in 90\% and no upsetting, confusing or difficult questions were identified by the study group. Although the suitability of question number 6 in the local context was raised in assessing the content validity only $22 \%$ of the sample had marked it as irrelevant.

\section{Discussion}

Health related quality of life measures have been used in parallel to clinical outcome measures in the field of Dermatology in the recent past. This necessitates a validated disease specific quality of life measuring instrument in the language used by a majority of the population.

During the translational process which ensured consistency with the original English version of the DLQI, only question 8 regarding the word "partner" showed a discrepancy. This question had given rise to difficulties in translation with its original English meaning even in other cultural settings $[10,11]$.

Although the suitability of question number 6 in the local context was raised in assessing the content validity, only $22 \%$ of the sample marked it as irrelevant. Therefore the authors feel the content of the DLQI should not be altered.

When compared with the SF-36, all domain correlations were above 0.4 which was sufficient for a moderate correlation. The results in this study showed better correlations with SF-36 than some other validation studies [10,11]. Physical and social functioning domains indicated better criterion validity compared to the vitality domain.

The DLQI (Sinhala) appear to discriminate well between the healthy and disease groups similar to its original version [5]. Furthermore, it discriminates quality of life changes in different diseases and within the disease groups according to their severity.

As the time taken to complete the DLQI (Sinhala) ranged from 3-5 minutes in the majority of the sample and the response rate was $98 \%$, it was proved to be a simple, practical, and acceptable quality of life instrument that can be used in the busy clinics in Sri Lanka. The rate of incorrectly marked questions was not significant except in question 7. This question was misunderstood by $22 \%$ but as the standard interpretation of incorrectly marked questions was used this did not affect the data adversely.

The mean DLQI score of the study population was 8.58. Most ethnic groups have a DLQI score of 6-10 [5,10- 
12]. Therefore the mean DLQI score of this study is consistent with the other ethnic groups.

\section{Conclusion}

The validated DLQI (Sinhala) questionnaire appears to be a reliable, practical and culturally acceptable quality of life measuring tool.

\section{Acknowledgements}

The authors would like to acknowledge Professor Andrew Finlay of Department of Dermatology, Cardiff University, Wales, United Kingdom for his valuable assistance in carrying out this study.

\section{References}

1. Ryan TJ. Disability in dermatology. British Journal of Hospital Medicine 1991; 46: 33-6.

2. Jowett S, Ryan TJ. Skin disease and handicap: an analysis of the impact of skin conditions. Social Sciences and Medicine 1985; 20:425-9.

3. Girishery IH, Link BG. Feeling of stigmatization in patients with psoriasis. Journal of the American Academy of Dermatology 1989; 20: 53-63.

4. Motley RJ, Finlay AY. How much disability is caused by Acne? Clinical and Experimental Dermatology 1989; 14: 194-8.
5. Finlay AY, Khan GK. Dermatology Life Quality Index (DLQI): a simple practical measure for routine clinical use. Clinical and Experimental Dermatology 1994; 19: 210-6.

6. Lewis VL, Finlay AY. Ten years experience of the Dermatology Life Quality Index (DLQI). Journal of Investigative Dermatology Symposium Proceedings 2004; 9: $169-80$.

7. Pete V, Jenkinson C, Fitzpatrick R. PDQ-39: a review of the development, validation and application of Parkinson's disease quality of life questionnaire and its associated measures. Journal of Neurology 1998; 245: 10-4.

8. Cronbach LJ, Meehl PE. Construct validity in psychological tests. Psychological Bulletin 1955; 52: 281-302.

9. Testa MA, Simonson DC. Assesment of quality of life outcomes. New England Journal of Medicine 1996; 334: 833-40.

10. Takahashi N, Suzukamo Y, Nakamura M, et al. Japanese version of the Dermatology Life Quality Index: validity and reliability in patients with acne. Health and Quality of Life Outcomes 2006; 4: 46.

11. Ozturkcan S, Ermertcan AT, Eser E, Sahin MT. Cross validation of the Turkish version of Dermatology Life Quality Index. International Journal of Dermatology 2006; 45: 1300-7.

12. Hahn HB, Melfi CA, Chuang TS, et al. Use of the Dermatology Life Quality Index (DLQI) in a Midwestern US urban clinic. Journal of the American Academy of Dermatology 2001; 45: 44-8. 